\section{Cureus}

\title{
Successful Treatment of Legionnaires' Disease with Tigecycline in an Immunocompromised Man with a Legion of Antibiotic Allergies
}

\author{
Michael Arget ${ }^{1}$, Justin Kosar ${ }^{2}$, Brandon Suen ${ }^{1}$, Shaqil Peermohamed ${ }^{3}$ \\ 1. Internal Medicine, University of Saskatchewan College of Medicine, Saskatoon, CAN 2. Miscellaneous, \\ Saskatchewan Health Authority, Saskatoon, CAN 3. Internal Medicine / Infectious Disease, University of \\ Saskatchewan College of Medicine, Saskatoon, CAN
}

Corresponding author: Shaqil Peermohamed, shaqil.peermohamed@saskhealthauthority.ca

\section{Abstract}

Legionella species are Gram-negative bacilli that are relatively rare causes of communityacquired pneumonia but can be associated with significant morbidity and mortality if unrecognized or improperly treated. Limited data exist regarding the use of tigecycline, a third generation glycylcycline, in the treatment of Legionnaires' disease. We present an immunocompromised patient with Legionnaires' disease and allergies to both fluoroquinolones and macrolides, which are first-line treatment options for Legionnaires' disease. He was successfully treated using tigecycline, a third generation glycylcycline, indicating that tigecycline may serve as a safe and effective alternative therapeuticl option for treatment of Legionnaires' disease.

Categories: Infectious Disease, Pulmonology

Keywords: legionella, legionnaires’ disease, pneumonia, tigecycline

\section{Introduction}

Legionnaires' disease is a relatively rare cause of community-acquired pneumonia caused by Legionella species; however, 20\%-25\% of patients who are hospitalized with Legionnaires' disease require invasive mechanical ventilation and average mortality rates for sporadic disease range from $10 \%$ to $15 \%$ [1]. Legionnaires' disease is caused by inhalation of Legionella species, which are intracellular, Gram-negative bacilli ubiquitously found in the environment [1-2]. Host risk factors for Legionnaires' disease include male gender, age older than 50 years, cigarette smoking, diabetes, end-stage renal failure, organ transplantation and immunosuppression, such as glucocorticoids or anti-rejection drugs following organ transplantation [1]. Travel is an important and underappreciated risk factor associated with legionellosis in a community setting [1]. Treatment options for Legionnaires' disease include macrolides, fluoroquinolones, or tetracycline; however, preferred therapies for immunocompromised patients with Legionnaires' disease include levofloxacin and azithromycin [1-3]. We describe an immunocompromised and severely ill patient with Legionnaires' disease and who also has allergies to both fluoroquinolones and macrolides; he was successfully treated using tigecycline, a third generation glycylcycline, indicating that tigecycline may serve as a safe and effective alternative therapeutic option for treatment of Legionnaires' disease in select cases. 


\section{Cureus}

\section{Case Presentation}

A 61-year-old Caucasian man presented to the emergency department in autumn with one week of dyspnea, productive cough, myalgia, and fever. He denied any chest pain or hemoptysis. His past medical history was significant for hypertension, diabetes mellitus, chronic kidney disease, and non-Hodgkin's lymphoma with receipt of an allogeneic stem cell transplant 13 years prior. Given prior complications due to graft versus host disease, he was receiving prednisone at a maintenance dose of $15 \mathrm{mg}$ daily for several years. He had multiple documented allergies to penicillin, sulfa drugs, macrolides and fluoroquinolones, with reported reactions including rash, hives, and anaphylaxis. Approximately one week prior to the onset of symptoms, he was traveling in the Midwest United States with his partner and staying in various hotels.

Upon arrival to the hospital, he was noted to have a heart rate of 130 beats per minute, a blood pressure of 128/76 $\mathrm{mmHg}$, a respiratory rate of 30 breaths per minute with an oxygen saturation of $89 \%$ requiring eight liters of supplementary oxygen, and an oral temperature of $39.8^{\circ} \mathrm{C}\left(103.6^{\circ} \mathrm{F}\right)$. He was in acute respiratory distress and had evident decreased breath sounds and crackles bilaterally. He was noted to have normal heart sounds without any murmurs, rubs, or gallops. He did not have any rash on examination.

Laboratory investigations revealed a normal peripheral leukocyte count of 10.4 x $10^{9}$ cells/L $\left(10.4 \times 10^{3}\right.$ cells $\left./ \mu \mathrm{L}\right)$, decreased hemoglobin of $110 \mathrm{~g} / \mathrm{L}(11.0 \mathrm{~g} / \mathrm{dL})$, decreased platelet count of $96 \times 109$ cells/L (96 x 10 $30^{3}$ cells/ $\left.\mu \mathrm{L}\right)$, and increased creatinine of $676 \mu \mathrm{mol} / \mathrm{L}(7.65 \mathrm{mg} / \mathrm{dL})$. His liver enzymes were normal. His initial chest radiograph revealed diffuse, bilateral air space opacities in the mid and lower lung zones (Figure 1).

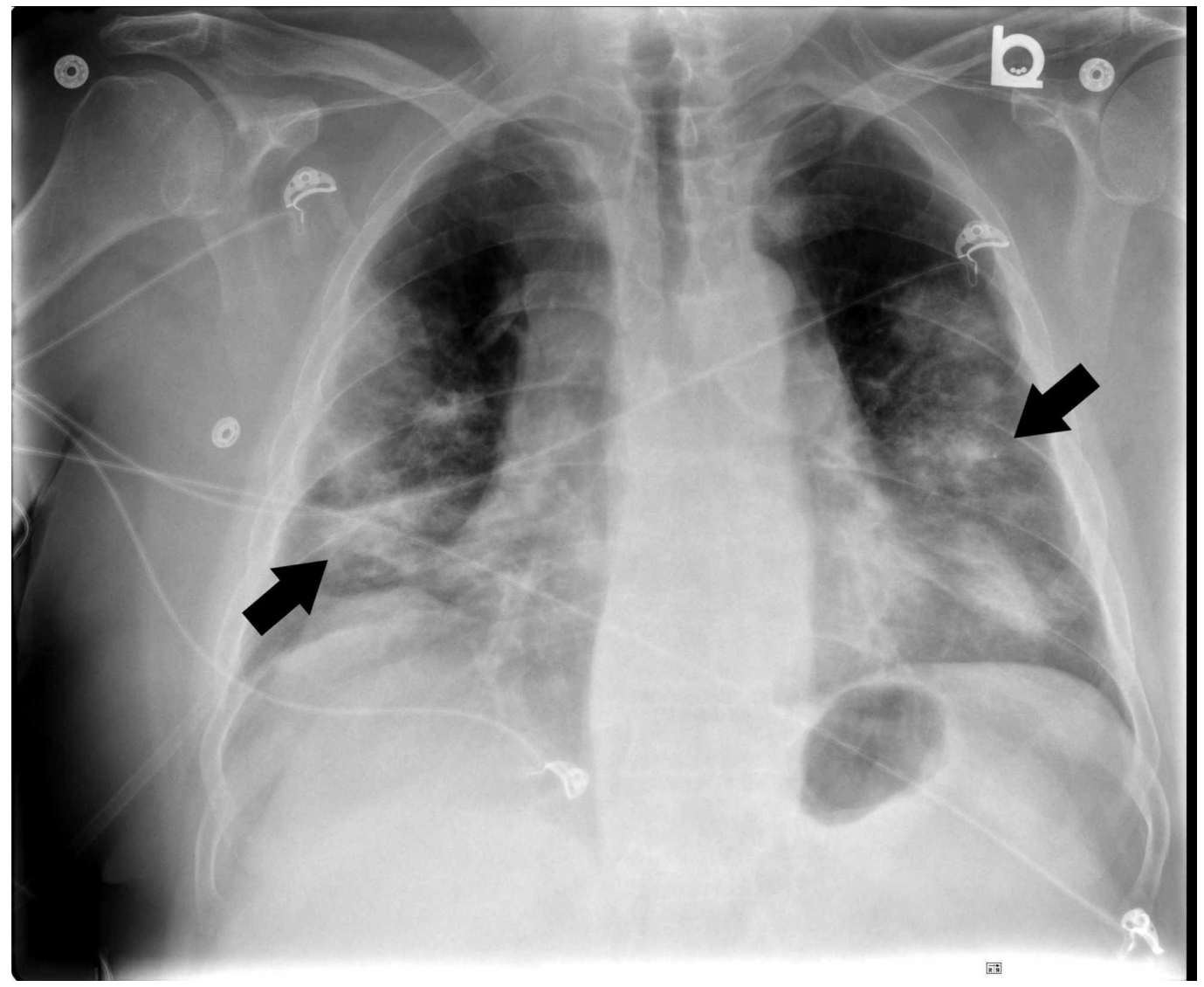

FIGURE 1: Chest radiograph on admission showing diffuse, 


\section{Cureus}

\section{bilateral air space opacities (arrows) in the mid and lower lung}

zones.

Two sets of blood cultures were collected and sputum samples were sent for culture as well as stains and polymerase chain reaction (PCR) testing for Pneumocystis jirovecii (P. jirovecii). A nasopharyngeal sample was collected for respiratory virus PCR testing for influenza A and B, respiratory syncytial virus $A$ and $B$, coronavirus, parainfluenza virus, rhinovirus, enterovirus, adenovirus, bocavirus, and metapneumovirus. In addition, a serum cytomegalovirus (CMV) PCR and Legionella urine antigen were sent. He was empirically started on meropenem, vancomycin, oseltamivir, and intravenous pentamidine.

Despite initiation of broad spectrum antimicrobials, he continued to deteriorate with increasing oxygen demands, persistent fever, hemodynamic instability, and worsening radiographic infiltrates (Figure 2).

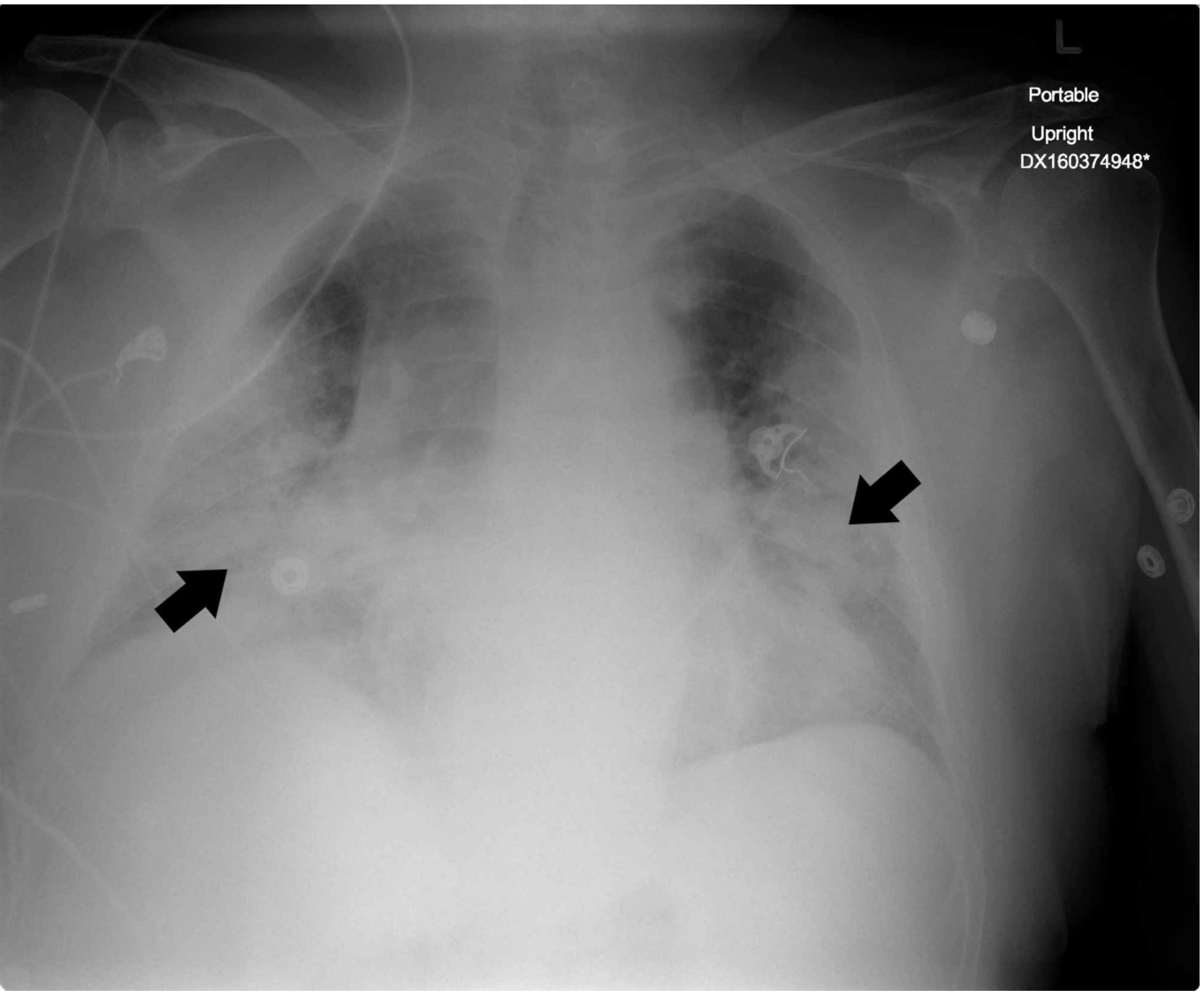

FIGURE 2: Chest radiograph $48 \mathrm{~h}$ following hospital admission, showing worsening bilateral pulmonary opacification (arrows).

Sputum and blood cultures were negative for any bacterial growth; sputum stains and PCR were negative for $P$. jirovecii. His serum CMV PCR was negative. His nasopharyngeal swab for respiratory virus testing was negative for influenza A and B, respiratory syncytial virus A and B, coronavirus, parainfluenza virus, rhinovirus, enterovirus, adenovirus, bocavirus, and metapneumovirus; however, his Legionella urine antigen was positive. 
According to his pharmacy records, he had previously received and tolerated a five-day course of moxifloxacin approximately one year prior to this hospital admission. Given his diagnosis of Legionnaires' disease, intravenous moxifloxacin therapy was initiated. However, shortly following receipt of his first dose of moxifloxacin, he developed an allergic reaction with a generalized, erythematous, maculopapular rash and angioedema, necessitating administration of epinephrine.

Given his documented allergies to both fluoroquinolones and macrolides, hemodynamic instability, and concern for poor gastrointestinal absorption of oral antimicrobials, he was subsequently treated with intravenous tigecycline with an initial, loading dose of $100 \mathrm{mg}$, followed by $50 \mathrm{mg}$ twice daily for a total of 14 days of therapy. Intravenous doxycycline is not readily available in our institution. His oxygen requirements decreased and fever resolved following 48 hours of treatment with tigecycline. All other antimicrobials were discontinued once the diagnosis of Legionnaires' disease was made. There was no recurrence of infection after three months of follow-up; his repeat chest radiograph showed resolution of his bilateral air space opacities.

Later in discussion with the North Dakota Department of Health and Centers for Disease Control and Prevention, it was determined that there was an ongoing outbreak of Legionnaires' disease associated with five cases over a 13-month period; all cases including our patient had stayed at the same hotel. Subsequent environmental testing of the hotel was negative, but this may have been impacted by a recent deep clean of the hotel's ventilation system.

\section{Discussion}

Legionnaires' disease and its causative pathogen were first recognized in 1977, following a common-source outbreak of severe pneumonia involving 221 people at an American Legion convention in Philadelphia, Pennsylvania in 1976 [1]. Outbreaks and clusters of cases of Legionnaires' disease have been associated with contaminated cooling towers, whirlpools, hospital decorative water fountains, hot spring spas, and water births [1-2].

Legionnaires' disease can be associated with a prodromal illness with symptoms including fever, headache, myalgia, and anorexia; however, the clinical presentation of Legionnaires' disease is often nonspecific and difficult to distinguish from other causes of communityacquired pneumonia [1-2]. Blood and sputum cultures are relatively insensitive in diagnosing Legionnaires' disease; in contrast, urine antigen testing has a sensitivity of $60 \%-95 \%$ and specificity greater than 99\% [1]. Urine antigen testing for Legionella only detects Legionella pneumophila serogroup 1 (Lp1) and is most sensitive for the detection of the Pontiac subtype of Lp1, which causes the majority of cases of community-acquired Legionnaires'

disease [1]. Legionella urine antigen testing will often be positive on the first day of illness and remain positive for several weeks [1]. Molecular testing of lower respiratory tract specimens can also be used to identify both Legionella pneumophila and Legionella species by PCR [1].

Preferred therapies for immunocompromised patients with Legionnaires' disease include levofloxacin and azithromycin [1-3]. Tigecycline is a third generation, intravenous glycylcycline and minocycline derivative that inhibits bacterial protein synthesis by binding to bacterial 30S ribosomal subunits [4]. Prior in vitro and animal model studies have shown that tigecycline achieves high intracellular concentrations [5]. However, demonstrated clinical effectiveness of tigecycline in the treatment of community-acquired pneumonia in humans with Legionnaires' disease remains limited.

Two prior case reports describe successful use of tigecycline in the treatment of immunocompromised patients with legionellosis; however, fluoroquinolones were used as initial therapy in both of these cases and tigecycline was later added to their antimicrobial 
regimen [6-7]. A recently published case series describes eight patients with Legionnaires' disease who were switched to tigecycline, often due to worsening sepsis and/or respiratory status, following initial exposure to macrolide and/or fluoroquinolone therapy (median of three days) [8]. All but one of these eight patients received combination therapy with tigecycline plus either levofloxacin or azithromycin as part of their treatment regimen once tigecycline was added. Furthermore, the one patient in this case series who received 14 days of tigecycline monotherapy had received eight days of azithromycin prior to switching therapy. Thus, it is difficult to ascertain whether clinical improvement in these cases was due to the addition of tigecycline or post-antibiotic effect and delayed response from fluoroquinolone/macrolide therapy.

Integrated results from two randomized controlled trials showed comparable cure rates between tigecycline and levofloxacin in the treatment of hospitalized patients with community-acquired pneumonia, of which a small proportion were diagnosed with Legionnaires' disease in each treatment arm [9]. While the integrated results of these two randomized controlled trials support the early use of tigecycline as empiric treatment of community-acquired pneumonia, one of these trials permitted switching to oral levofloxacin following at least three days of intravenous therapy if evidence of clinical improvement.

Current evidence, albeit limited, suggests that tigecycline may be added as combination therapy in severe cases of Legionnaires' disease. This case, however, demonstrates that tigecycline can be effective as a second-line treatment option for Legionnaires' disease in the setting of allergies to traditional mainstays of therapy. In 2013, the Food and Drug Administration (FDA) approved a new boxed warning about the higher risk of death among patients receiving tigecycline compared with other antibiotics, particularly apparent for hospital-acquired pneumonia and ventilator-associated pneumonia [10]. While both the FDA and Health Canada have approved tigecycline for treatment of community-acquired bacterial pneumonia, complicated skin and soft tissue infections, and complicated intra-abdominal infections, its use should be reserved for situations when alternative treatments are not suitable [10-11].

\section{Conclusions}

Legionnaires' disease is a rare cause of community-acquired pneumonia but can be associated with significant morbidity and mortality, especially amongst immunocompromised individuals. Although the evidence regarding the use of tigecycline in treating Legionnaires' disease is limited, this case report provides evidence supporting the use of tigecycline as a second-line therapeutic option in select cases where fluoroquinolone or macrolide therapy may be contraindicated.

\section{Additional Information \\ Disclosures}

Human subjects: Consent was obtained by all participants in this study. Conflicts of interest: In compliance with the ICMJE uniform disclosure form, all authors declare the following:

Payment/services info: All authors have declared that no financial support was received from any organization for the submitted work. Financial relationships: All authors have declared that they have no financial relationships at present or within the previous three years with any organizations that might have an interest in the submitted work. Other relationships: All authors have declared that there are no other relationships or activities that could appear to have influenced the submitted work.

\section{Acknowledgements}

We would like to acknowledge Ms. Jill Baber, Respiratory \& Syndromic Surveillance 
Epidemiologist with the North Dakota Department of Health in providing details pertaining to this outbreak of legionellosis.

\section{References}

1. Edelstein PH, Roy CR: Legionnaires' disease and pontiac fever. Mandell, Douglas, and Bennett's Principles and Practice of Infectious Diseases (Eighth Edition). Bennett JE, Raphael D, Blaser MJ (ed): Saunders, Philadelphia; 2015. 2:2633-2644.

2. Fields BS, Benson RF, Besser RE: Legionella and legionnaires' disease: 25 years of investigation. Clin Microbiol Rev. 2002, 15:506-526. 10.1128/CMR.15.3.506-526.2002

3. Pedro-Botet ML, Yu VL: Treatment strategies for legionella infection. Expert Opin Pharmacother. 2009, 10:1109-1121. 10.1517/14656560902900820

4. Moffa M, Brook I: Tetracyclines, glycylcyclines, and chloramphenicol. Mandell, Douglas, and Bennett's Principles and Practice of Infectious Diseases (Eighth Edition). Bennett JE, Raphael D, Blaser MJ (ed): Saunders, Philadelphia; 2015. 1:322-338.

5. Edelstein PH, Weiss WJ, Edelstein MA: Activities of tigecycline (GAR-936) against legionella pneumophila in vitro and in guinea pigs with legionella pneumophila pneumonia. Antimicrob Agents Chemother. 2003, 47:533-540.

6. Galstian GM, Drokov MIu, Katrysh SA, et al.: A case of legionellesis pneumonia verified by isolation of legionella pneumophila serogroup 1 from bronchoalveolar lavage fluid treated with levofloxacine and tigecycline. Ter Arkh. 2011, 83:61-65.

7. Valve K, Vaalasti A, Anttila VJ, Vuento R: Disseminated legionella pneumophila infection in an immunocompromised patient with tigecycline. Scand J Infect Dis. 2010, 42:152-155. 10.3109/00365540903359895

8. Slawek D, Altshuler D, Dubrovskaya Y, Louie E: Tigecycline as a second-line agent for legionnaires’ disease in severely ill patients. Open Forum Infect Dis. 2017, 4:ofx184. 10.1093/ofid/ofx184

9. Tanaseanu C, Bergallo C, Teglial O, et al.: Integrated results of 2 phase 3 studies comparing tigecycline and levofloxacin in community-acquired pneumonia. Diagno Microbiol Infect Dis. 2008, 61:329-338. 10.1016/j.diagmicrobio.2008.04.009

10. Dixit D, Madduri RP, Sharma R: The role of tigecycline in the treatment of infections in light of the new black box warning. Expert Rev Anti Infect Ther. 2014, 12:397-400.

$10.1586 / 14787210.2014 .894882$

11. Tygacil (tigecycline). (2018). Accessed: March 25, 2019: https://www.pfizer.ca/tygaciltigecycline. 